\title{
Viral Proteases as Targets for Coronavirus Disease 2019 Drug Development
}

\author{
Wei Zhu, Zeenat Shyr, Donald C. Lo, and Wei Zheng \\ National Center for Advancing Translational Sciences, National Institutes of Health, Bethesda, Maryland \\ Received April 22, 2021; accepted May 6, 2021
}

\section{ABSTRACT}

Coronavirus disease 2019 (COVID-19), caused by severe acute respiratory syndrome coronavirus 2 (SARS-CoV-2), continues to be a global threat since its emergence. Although several COVID-19 vaccines have become available, the prospective timeframe for achieving effective levels of vaccination across global populations remains uncertain. Moreover, the emergence of SARS-CoV-2 variants presents continuing potential challenges for future vaccination planning. Therefore, development of effective antiviral therapies continues to be an urgent unmet need for COVID-19. Successful antiviral regimens for the treatment of human immunodeficiency virus and hepatitis $C$ virus infections have established viral proteases as validated targets for antiviral drug development. In this context, we review protease targets in drug development, currently available antiviral protease inhibitors, and therapeutic development efforts on SARS-CoV-2 main protease and papain-like protease.

\section{SIGNIFICANT STATEMENT}

Coronavirus disease 2019 (COVID-19) continues to be a global threat since its emergence. The development of effective antiviral therapeutics for COVID-19 remains an urgent and long-term need. Because viral proteases are validated drug targets, specific severe acute respiratory syndrome coronavirus 2 protease inhibitors are critical therapeutics to be developed for treatment of COVID-19.

\section{Introduction}

Severe acute respiratory syndrome coronavirus 2 (SARS$\mathrm{CoV}-2$ ) is a member of the Coronaviridae family and Betacoronavirus genus. This virus first emerged in late 2019 and causes the deadly coronavirus disease 2019 (COVID-19). To combat COVID-19, SARS-CoV-2 vaccines have been developed with unprecedented speed and with already three having received emergency use authorization from the US Food and Drug Administration (FDA) at the time of this writing. However, mutations in the spike protein of SARS-CoV-2 have led to the emergence of several rapidly spreading variants, such as those originally discovered in the United Kingdom, South Africa, and Brazil; such continuing strain variance may become a threat to the effectiveness of current COVID-19 vaccine strategies (www.cdc.gov/coronavirus/2019-ncov/more/ science-and-research/scientific-brief-emerging-variants.html).

Moreover, increasing success of vaccination programs across global populations may increase selection pressure for the

Financial support for this work was provided by the Intramural Research Program of the National Institutes of Health National Center for Advancing Translational Sciences.

No author has an actual or perceived conflict of interest with the contents of this article.

https://dx.doi.org/10.1124/jpet.121.000688. emergence of new SARS-CoV-2 variants. In contrast, although substantial efforts have been undertaken to develop antiviral therapeutics to treat COVID-19, to date only remdesivir (an RNA-dependent RNA polymerase inhibitor) and two monoclonal antibody cocktails have received emergency use authorization from FDA, with the effectiveness of remdesivir remaining uncertain in late-stage disease (Pan et al., 2021). Thus, the development of effective antiviral therapeutics for COVID-19 remains an urgent and long-term need.

SARS-CoV-2 is a single-strand positive-sense RNA virus comprising an RNA genome and four structural proteins: nucleocapsid protein, membrane protein, envelope protein, and spike glycoprotein (S-protein). The $5^{\prime}$ portion of the SARSCoV-2 RNA genome contains two overlapping open reading frames $(O R F s), O R F 1 a$ and $O R F 1 b$, which are translated into two large polyproteins that are cleaved by viral proteases to produce a series of nonstructural proteins (NSPs) (Fig. 1). The NSPs of SARS-CoV-2 play essential roles in viral replication. Among the SARS-CoV-2 NSPs, there are two proteases: 3-chymotrypsin-like "main" protease (3CLpro, also known as Mpro) and papain-like protease (PLpro, the protease domain of NSP3). These two proteases act on different sites in the polyproteins and are responsible for cleavage of the viral polypeptides (Fig. 1). Thus, inhibition of one or both viral proteases

ABBREVIATIONS: ACE2, angiotensin-converting enzyme 2; AMC, 7-amino-4-methylcoumarin; 3CLpro, 3-chymotrypsin-like protease; COVID-19, coronavirus disease 2019; FDA, Food and Drug Administration; FLuc, firefly luciferase; FRET, fluorescence resonance energy transfer; HCV, hepatitis C virus; HIV, human immunodeficiency virus; HTS, high-throughput screening; ISG15, interferon-stimulated gene 15 protein; L, Large BiT; NSP, nonstructural protein; ORF, open reading frame; PLpro, papain-like protease; RdRp, RNAdependent RNA polymerase; S, Small BiT; SARS-CoV, severe acute respiratory syndrome coronavirus; SARS-CoV-2, severe acute respiratory syndrome coronavirus 2; S-protein, spike glycoprotein. 
should effectively halt the production of SARS-CoV-2 NSPs, thereby inhibiting viral replication in host cells.

Proteases are enzymes that hydrolyze the specific amide bonds of peptide units in polypeptides and proteins. They are classified into seven broad groups: serine, cysteine, threonine, aspartic, glutamic, metallo, and asparagine proteases based on their principal catalytic residues. Human cells have many proteases that are critical for important biologic functions and also have pathologic roles in many diseases such as cancer (López-Otín and Matrisian, 2007). Here, we discuss the function of viral proteases in viral life cycle, FDA-approved protease inhibitors, protease assay development, drug repurposing efforts targeting viral proteases, and prospective therapeutic development for SARS-CoV-2.

\section{Viral Protease Function in the Viral Life Cycle}

Both host proteases and viral proteases play key roles in the viral infection and replication processes in human cells. The S-proteins of SARS-CoV-2 bind to angiotensin-converting enzyme 2 (ACE2) receptors in host cells to initiate cell entry. Host proteases such as cell surface transmembrane protease serine 2 facilitate the binding of SARS-CoV-2 S-protein to ACE2 receptor with high affinity and efficient fusion of viral membrane to host lipid membrane through S-protein priming (Shang et al., 2020). Once SARS-CoV-2 enters the host cells, it exploits the host cell machinery to translate $O R F$ s of viral RNA. The RNA genome of SARS-CoV-2 is approximately 30 kilobase with 14 ORFs. ORF1a and ORF1b are translated into the polyproteins pp1a and pp1ab, which are cleaved into 16 NSPs by the two viral proteases included in these two polyproteins (Gordon et al., 2020).

3CLpro hydrolyzes no fewer than 11 cleavage sites in the polyproteins to produce a series of NSPs, including itself (NSP5) and other critical viral proteins like RNA-dependent RNA polymerase (RdRp, NSP12) and helicase (NSP13) (Fig. 1)
(Alexander et al., 2020). It recognizes the sequence of Leu-Gln $\downarrow$ (Ser, Ala, Gly) at most sites, in which $\downarrow$ represents the cleavage site (Zhang et al., 2020). 3CLpro is a three-domain (domains I-III) cysteine protease that is highly conserved among coronaviruses. SARS-CoV-2 3CLpro has 95\% sequence identity and a highly similar three-dimensional structure with 3CLpro of SARSCoV (Zhang et al., 2020). Coronavirus 3CLpro features a nonclassic Cys-His catalytic dyad in the cleft between domains I and II (Anand et al., 2003; Yang et al., 2003; Dai et al., 2020). The proteolytic cleavage process starts by liberating itself from ppla and pp1ab at its own N and C termini (Hsu et al., 2005; Lee et al., 2020), followed by maturation and active dimer formation. Discovery of small-molecule inhibitors that interrupt this initial autocleavage event would be difficult, as intramolecular reactions are favored and the early-stage intermediate has yet to form a mature and stable active site (Chen et al., 2010; Steuten et al., 2021).

Coronavirus PLpro performs cleavage on NSP1, NSP2, and NSP3 at the N-terminal region of polyproteins by recognizing the tetrapeptide Lys-Xxx-Gly-Gly motif located in between NSP1 and NSP2, NSP2 and NSP3, and NSP3 and NSP4 (Fig. 1) (Harcourt et al., 2004; Gioia et al., 2020; Rut et al., 2020). PLpro is part of the multidomain protein NSP3, which is the largest individual protein (not including polyproteins) encoded by coronavirus genome with a molecular mass of $\sim 200 \mathrm{kD}$ (Lei et al., 2018). Along with the PLpro domain, NSP3 possesses several other domains, including a ubiquitin-like domain 1, a Glu-rich acidic domain, a macrodomain, a ubiquitin-like domain 2, an NSP3 ectodomain, domains Y1 and CoV-Y of unknown functions, and transmembrane region 1 and transmembrane region 2 the two transmembrane regions TM1 and TM2 (Lei et al., 2018). Although SARS-CoV-2 PLpro shares $83 \%$ sequence identity with SARS-CoV PLpro, they prefer different host substrates (Rut et al., 2020; Shin et al., 2020). In host cells, SARS-CoV-2 PLpro predominantly targets the ubiquitin-like interferon-stimulated gene 15 protein (ISG15), whereas SARS-CoV PLpro preferentially cleaves ubiquitin chains (Shin et al., 2020).

\section{Genome position(bp)}

\begin{tabular}{lllll}
\hline \hline 0 & 10,000 & 15,000 & 20,000 & 25,000
\end{tabular}

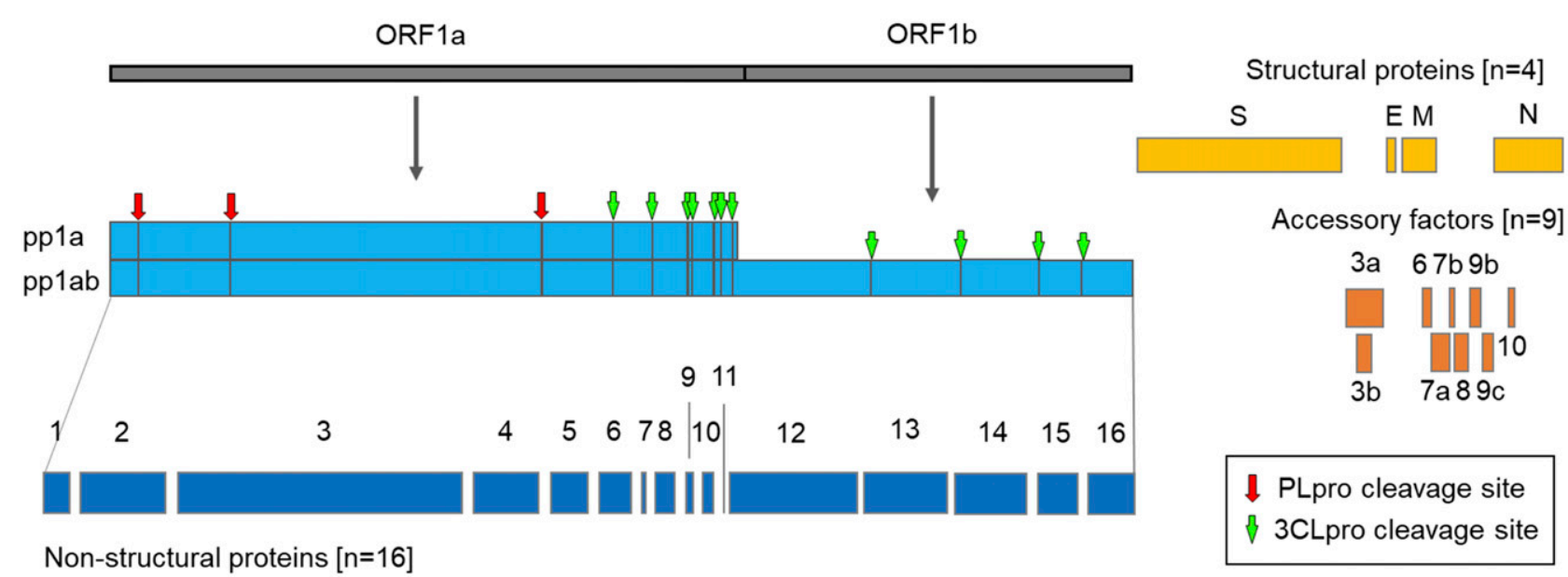

Fig. 1. Scheme of SARS-CoV-2 genome. ORF1a and ORF1b occupying two-thirds of the 30-kb RNA genome directly translate to 2 polyproteins, pp1a and pp1ab. The polyproteins are processed to produce 16 nonstructure proteins by 3CLpro and PLpro. Other ORFs encode four structural proteins and nine accessory factors of SARS-CoV-2. E, envelope protein; M, membrane protein; N, nucleocapsid protein. 
Besides cleaving polyproteins, SARS-CoV PLpro was found to harbor two other proteolytic activities, removal of ubiquitin and ubiquitin-like protein ISG15 from cellular proteins (Rut et al., 2020). SARS-CoV-2 PLpro features the same deubiquitinating and deISGylating activities as SARS-CoV PLpro (Shin et al., 2020). Through the deubiquitinating and de-ISGylating activities in host cells, SARS-CoV PLpro inhibits host innate immune responses that are initially mounted as an inflammatory response and subsequently turned to an interferon response (Klemm et al., 2020). The interferon response involves the transcriptional upregulation of over 300 interferon-stimulated genes that endows host cells with the capability to detect and respond to viral threats (Ivashkiv and Donlin, 2014). Infection of SARS-CoV-2 in humans leads to dysregulated inflammatory responses, which contributes to substantial morbidity and mortality (Berlin et al., 2020). Targeting SARSCoV-2 PLpro may thus not only suppress SARS-CoV-2 infection but also allow fuller antiviral host immune response.

\section{Viral Protease Inhibitors}

FDA-Approved Protease Inhibitors. Protease inhibitors were explored to combat COVID-19 upon onset of the outbreak, with numerous efforts focusing on repurposing drugs that had previously been approved for other diseases, such as human immunodeficiency virus (HIV) and hepatitis $\mathrm{C}$ virus (HCV). HIV and HCV viral proteases mediate the cleavage of polyprotein precursors at different sites, which results in mature individual proteins (Lin, 2006; Ghosh et al., 2016). Protease inhibitors act as peptidomimetics that compete at the active substrate binding sites of proteases, thereby preventing cleavage of viral polyproteins.

Saquinavir was the first approved protease inhibitor for HIV (De Clercq and Li, 2016). Saquinavir, along with eight other approved HIV protease inhibitors-ritonavir, indinavir, nelfinavir, amprenavir, fosamprenavir, lopinavir, atazanavir, and darunavir-share similar mechanisms of action in which a hydroxyethylene bond serves as the peptidomimetic scaffold (De Clercq and Li, 2016). Tipranavir is the only exception, which bears the coumarin scaffold (De Clercq, 2013).

The HCV serine protease non-structural protein 3 (NS3) requires non-structural protein $4 \mathrm{~A}(\mathrm{NS} 4 \mathrm{~A})$ as a cofactor for optimal activity (Lin, 2006). HCV NS3/4A protease inhibitors are peptidomimetics that interact with the active serine site. Antiviral inhibitors such as telaprevir and boceprevir are linear peptidomimetics that form reversible covalent adducts with the enzyme (Bartenschlager et al., 2013). Both telaprevir and boceprevir have been discontinued for commercial reasons (De Clercq and Li, 2016). Other currently available anti-HCV protease drugs such as faldaprevir, grazoprevir, paritaprevir, simeprevir, and voxilaprevir are linear peptidomimetics or macrocyclic inhibitors that bind noncovalently with their targets (Bartenschlager et al., 2013).

The combination of lopinavir with ritonavir, both approved protease inhibitors for HIV, was among the earliest clinical trials to treat COVID-19. During the SARS outbreak in 2003, a combination of lopinavir combined with ritonavir was shown to reduce overall death rate and intubation time (Chan et al., 2003; Yao et al., 2020). Generally, lopinavir is metabolized rapidly in vitro, and ritonavir is commonly coadministered with other protease inhibitors because ritonavir boosts the plasma levels of other protease inhibitors and increases their half-lives and bioavailability by inhibiting the major cytochrome
P450 isoforms CYP3A4 and CYP2D6 (Wensing et al., 2010). Coadministration of lopinavir with ritonavir can increase its area under the drug plasma concentration-time curve by over 100-fold (Hurst and Faulds, 2000). Although such high plasma exposures can be achieved, the combination of lopinavir and ritonavir showed no clinical benefit in treating patients with severe COVID-19 compared with standard of care (Cao et al., 2020).

In addition to the clinical trials mentioned above, many preclinical studies have tested the efficacy of existing FDAapproved protease inhibitors against SARS-CoV-2 as well. Lo et al. (2021) found that simeprevir significantly reduced SARS-CoV-2 viral load in vitro by inhibiting 3CLpro and unexpectedly also suppressed RdRp activity. It was also found that simeprevir can modulate host immune responses and can synergize with remdesivir, improving antiviral activity in vitro. In another study that combined virtual screening and experimental testing, approved protease inhibitors, boceprevir, telaprevir, and nelfinavir showed varied activities in inhibiting SARS-CoV-2 protease and viral replication (Pathak et al., 2021). Jan et al. (2021) also found that boceprevir and nelfinavir mesylate can inhibit SARS-CoV-2 3CLpro activity. Although these approved protease inhibitors have demonstrated activity against SARS-CoV-2 in vitro, they have yet to be investigated clinically.

Novel Protease Inhibitors for SARS-CoV-2. Numerous efforts to develop novel protease inhibitors for COVID-19 have been actively carried out in the past year. Following the elucidation of X-ray structure of unliganded SARS-CoV-2 3CLpro, Zhang et al. (2020) developed a lead compound $13 \mathrm{~b}$ with $\mathrm{IC}_{50}$ of $0.67 \pm 0.18 \mu \mathrm{M}$ against recombinant SARS-CoV-2 3CLpro. The pharmacokinetic characterization of $13 \mathrm{~b}$ revealed a pronounced lung tropism when administrated via inhalation to mice. Besides the unliganded structure, the liganded complex of SARS-CoV-2 $3 \mathrm{CLpro}$ has also been used as a model to assist new drug discovery. Jin et al. (2020) found that N3, a Michael acceptor inhibitor, showed antiviral activity against SARS-CoV-2 in vitro (Yang et al., 2005). They cocrystallized the structure of SARS-CoV-2 3CLpro in complex with N3, which offered an in silico model for identifying lead compounds to target SARS-CoV-2 3CLpro. Through a combination of virtual and high-throughput screening (HTS), ebselen was identified that showed a $\mathrm{IC}_{50}$ value of $0.67 \pm$ $0.09 \mu \mathrm{M}$ in enzymatic assay and half-maximal $\mathrm{EC}_{50}$ value of $4.67 \pm 0.80 \mu \mathrm{M}$ in a cell-based SARS-CoV-2 cytopathic effect assay. In another study of dipeptidyl compounds in complex with coronavirus 3CLpro, Rathnayake et al. (2020) designed a new dipeptidyl series that showed varying degrees of activity against coronavirus 3CLpro in enzyme assays. Two optimal compounds exhibited antiviral effects against SARS-CoV-2 infection in primary human airway epithelial cells in vitro. One lead compound improved survival rate to $100 \%$ in a mouse model of Middle East respiratory syndrome coronavirus (MERS-CoV) infection, along with reduced lung viral load and lung histopathology (Rathnayake et al., 2020).

Crystallographic structure of the ligand-protease complex also facilitated the discovery of another 3CLpro inhibitor, PF00835231 (metabolite of PF-0730481), which is currently being tested in a COVID-19 clinical trial (ClinicalTrials.gov identifier: NCT04535167). PF-00835231 was originally identified by a research effort focused on discovering ketone-based inhibitors of SARS-CoV 3CLpro through employing ligand-protease structures solved by X-ray crystallography (Hoffman et al., 2020). Preclinical studies revealed that PF-00835231 is a potent 
antiviral agent against SARS-CoV-2 3CLpro with inhibitory constant $\left(K_{\mathrm{i}}\right)$ value in the nanomolar range in a biochemical assay. Its preclinical safety profile and absorption, distribution, metabolism, and excretion properties supported its progression to clinical evaluation (Boras et al., 2020). In a study by de Vries et al. (2020) PF-00835231 showed slight but statistically more potency than remdesivir in human hypotriploid alveolar basal epithelial cells expressing ACE2 exogenously, and both potently inhibited SARS-CoV-2 infection in human polarized airway epithelial cultures.

The cocrystal structures of SARS-CoV-2 3CLpro with approved drugs have also been used to design novel protease inhibitors. In a report by Qiao et al. (2021), the crystal structure of SARS-CoV-2 and its cocrystal structures with boceprevir and telaprevir were used to design and synthesize 32 protease inhibitors. All the synthesized compounds showed very potent activity against SARS-CoV-2 3 CLpro, with $\mathrm{IC}_{50}$ values ranging from 7.6 to $748.5 \mathrm{nM}$. Importantly, two compounds (MI-09 and MI-30) presented excellent antiviral activities in cell-based assays as well as in reducing lung viral load in mouse models when administrated orally or intraperitoneally (Qiao et al., 2021).

In comparison with 3CLpro, PLpro has been less well characterized, and its study is still in early stages with challenges (Gioia et al., 2020; Luan et al., 2020). As a viral protease, it remains an attractive drug target for SARS-CoV-2 (Maiti, 2020). Although only a few crystal structures of SARS-CoV-2 PLpro and/or complexed with ligands have been released, several in silico studies have been launched to identify potential inhibitors (Pitsillou et al., 2020; Huynh et al., 2021; Jamalan et al., 2021). In addition, a series of noncovalent small molecules that were originally designed for SARS-CoV have also shown potent inhibitory effects against SARS-CoV-2 (BáezSantos et al., 2015; Klemm et al., 2020). Klemm et al. (2020) resynthesized racemic forms of three SARS-CoV PLpro inhibitors, rac3j, rac3l, and rac5c, that exhibited low or submicromolar $\mathrm{IC}_{50}$ values against recombinant SARS-CoV-2 PLpro. In a SARS-CoV-2 cytopathic effect assay, these compounds partially rescued Vero E6 cells from SARS-CoV-2 infection. Dual inhibitors of 3CLpro and PLpro have also been reported. Two FDA-approved drugs, levothyroxine and manidipine-2HCl, were shown to inhibit both SARS-CoV-2 3CLpro and PLpro activities in vitro (Kuo et al., 2021).

\section{Screening Assays for Protease Inhibitors}

HTS assay development targeting proteases has been an ongoing effort for many years, with recent endeavors adapting these assays for discovery of SARS-CoV-2 protease inhibitors. Proteases recognize specific cleavage sites in substrates (proteins or polypeptides). Both biochemical and cell-based protease assays have been developed using specific substrate sequences of viral proteins to determine the activities of SARS-CoV-2 protease inhibitors.

Biochemical Assays. Fluorogenic substrates for hydrolytic enzymes have been extensively used in biochemical assays for HTS. In many cases, the donor fluorophore and an acceptor quencher are linked directly on opposite sides of a peptide chain containing a specific cleavage site that can be recognized by hydrolytic enzymes. The donor fluorophore and accepter quencher can undergo a process of fluorescence resonance energy transfer (FRET). In FRET, photons from the energetically excited donor fluorophore raise the energy state of electrons in the acceptor quencher to higher vibrational levels of the excited singlet state, resulting in the return of the donor fluorophore energy level to ground state without fluorescence emission (Kuo et al., 2004; Marras, 2006). FRET only occurs when the proximity of the donor and acceptor molecules lies in a specified range, usually within 10-100 $\AA$ (Haugland et al., 1969). In addition, the emission spectrum of the donor must overlap the absorption spectrum of the acceptor, and their transition dipole orientations must be approximately parallel. FRET efficiency dramatically falls as the distance between donor and acceptor exceeds a specific range-in the present case when the substrate is cleaved by the protease.

Quenched fluorescent peptides are widely used as substrates in enzymatic assays. A classic pair of quencher and fluorescence donor used for SARS-CoV-2 protease assays is DABCY and EDANS, respectively, which are linked to two ends of peptide substrate (Kuo et al., 2004; Zhu et al., 2020). In such applications, the presence of hydrolytic enzyme leads to the cleavage of peptide substrate. This significantly increases the distance between donor and acceptor molecules, resulting in loss of fluorescence quenching effect of the acceptor molecule and an increase in fluorescence signal (Fig. 2A).

Some fluorophores do not need specific acceptor quenchers, as their fluorescence is quenched when they are covalently linked to peptide substrates. For instance, the fluorophore 7amino-4-methylcoumarin (AMC) is typically linked to a peptide through formation of an amide bond that serves as a substrate cleavage site for proteases. Proteolysis of this amide bond liberates free AMC, leading to a significant increase in fluorescence that can be detected at a $342 \mathrm{~nm} / 441 \mathrm{~nm}$ excitation/emission wavelength. Breidenbach et al. (2021) synthesized such a fluorogenic substrate containing a C-terminal AMC moiety. The design of its structure, Boc-Abu-Tle-Leu-Gln-AMC, was based on the preference of 3CLpro for glutamine at its P1 position and an optimized P2 to P4 sequence (Rut et al., 2021). They found that the kinetic parameters of known 3CLpro inhibitors determined by the assay using Boc-Abu-Tle-Leu-Gln-AMC substrate are comparable to those from experiments with the DABCYL-EDANS substrate, indicating feasibility of this AMC substrate for HTS.

Such fluorogenic substrates are very amenable to use in HTS protease assays for the identification of inhibitors from

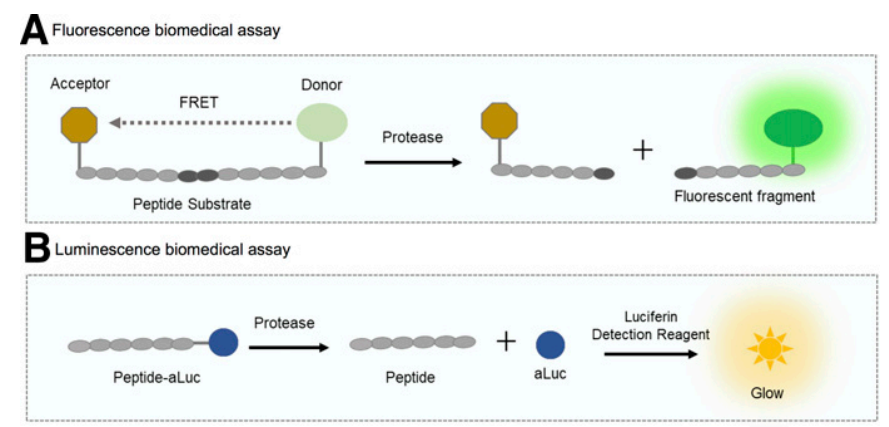

Fig. 2. Biochemical assays for identification of protease inhibitors. (A) In fluorescence assay, donor fluorescence in the substrate is quenched by an acceptor in intact peptide. The presence of protease cleaves the peptide and releases a high-fluorescence fragment. (B) The substrate in a luminescence assay has an aLuc linked to a peptide, which is inactive in the substrate. The cleavage under protease releases aLuc, which can further elicit glow in the presence of luciferin detection reagent. 
synthetic chemical and natural product libraries by supporting homogeneous assay format in which reagents are added in the assay protocol followed by readout without need for well/plate washing steps. However, compounds with intrinsic fluorescence or quenching effects that overlap the fluorophore emission or absorbance spectrum of the substrates can interfere with the assay signals. Thus, a secondary assay with different fluorescence emission spectrum or different detection methods should always be done to confirm initial hits. Recently, a luminescence assay has been reported with a peptide aminoluciferin (peptide-aLuc) substrate, which produces aLuc in the presence of 3CLpro. The aLuc can further initiate and drive a reaction with luciferase in the reagent mixture to produce luminescence light proportional to protease activity (Fig. 2B).

The peptide substrates used in HTS are designed to mimic the sequences of cleavage sites of 3CLpro and PLpro within the polyproteins. It is worth noting that the substrate specificities of 3CLpro and PLpro to their own cleavage sequences are distinct. Kuo et al. (2021) investigated the kinetics and substrate specificities of 3CLpro and PLpro to their corresponding sequences in the polyproteins representing the 11 and 3 cleavage sites, respectively. It was found that 3CLpro cleaved its own N-terminal cleavage site most efficiently, and the fastest rate of PLpro cleavage was obtained from the N-terminal cleavage site of NSP3. A SARS-CoV-2 3CLpro enzymatic assay using the substrate peptide resembling the most efficient 3CLpro cleavage sequence has been employed for drug repurposing screens, resulting in identification of several 3CLpro inhibitors in micromolar to submicromolar range (Zhu et al., 2020).

Of note, lead molecules identified in biochemical assays may not show antiviral activity in cell-based assays. First, compound permeability across the cell membrane is not addressed in biochemical assays. Compounds would become much weaker or inactive in cell-based assays if their cell membrane permeability is poor. Second, in cell-based assays, compounds may be metabolized or inactivated by cellular metabolism enzymes before they gain access to the drug target, causing significant reduction in net inhibitory activity. Third, drug efflux pumps on cell membranes can transport compounds out of the cells if the compounds prove to be substrates. Fourth, purified recombinant proteins used in biochemical assay may be folded improperly or miss subunits/cofactors that are needed for enzyme function in host cells, which can alter the observed activities of protease inhibitors identified in biochemical enzyme assays in subsequent cell-based enzyme assays.

Cell-Based Enzyme Assays. Cell-based enzyme assays are an essential follow-on to biochemical assays for reasons discussed above. Cell-based protease assays can be generated by cotransfecting a protease gene and a substrate reporter construct into the cell types of interest. To date, several cellbased assays with fluorescence and luminescence reporters have been developed for SARS-CoV-2 proteases. One such assay used GFP-derived protein (FlipGFP) as a reporter, in which fluorescence can be detected after FlipGFP is cleaved by 3CLpro (Froggatt et al., 2020). In FlipGFP, the 10th and 11th GFP $\beta$-strands ( $\beta 10-11)$ are expressed separately from the rest of the GFP $\beta$-barrel ( $\beta 1$-9) and are inactive in a parallel conformation when connected by a linker containing a 3CLpro cleavage site. When 3CLpro is expressed in cells, the linker is cut, allowing GFP $\beta 11$ to reorient antiparallel to $\beta 10$, which enables them to fit into the main GFP $\beta$ 1-9 barrel. As a result, fluorescence intensity in the presence of 3CLpro is increased by $~ 100$-fold over background (Fig. 3A) (Zhang et al., 2019; Froggatt et al., 2020).

In a study by Rawson et al. (2021), NanoBiT was employed as a luminescence reporter in a SARS-CoV-2 3CLpro cellbased assay. NanoBiT is engineered from NanoLuc luciferase and comprises two protein fragments: Large BiT (L, 17.6 kDa) and Small BiT (S, $1.3 \mathrm{kDa}$ ) (Dixon et al., 2016). Complementation occurs when $\mathrm{L}$ and $\mathrm{S}$ fragments are in close proximity to form a functional luciferase protein, whereas the $\mathrm{L}$ and $\mathrm{S}$ individual fragments do not have the luciferase function. The $\mathrm{L}$ and S fragments are connected by a 3CLpro cleavable linker that is coexpressed with SARS-CoV-2 3CLpro in HEK293T cells using a lentiviral vector (Fig. 3B). Lentiviral vector transfected cells do not have high luminescent signal, as 3CLpromediated cleavage of the L-S linkage site results in low luciferase activity. When inhibitors of 3CLpro block the cleavage of $\mathrm{L}$ and $\mathrm{S}$ fragments in the cells, luciferase activity increases, and a luminescent signal is detected. Notably, because 3CLpro inhibitors increase the luminescent signal, cytotoxic compounds will not produce false-positive hits in this cell-based assay.

The luciferase complementation methodology has also been used in developing cell-based assays to screen SARS-CoV-2 PLpro inhibitors. Instead of using NanoLuc luciferase, Smith et al. (2020) employed a firefly luciferase (FLuc) reporter in the PLpro cell-based assay (Fig. 3C). The reporter gene (substrate) was composed of four linked parts, including DnaE, a C-terminal FLuc fragment, a peptide with PLpro cleavage site, and an N-terminal FLuc fragment. The cleavage peptide sequence in this substrate resembles the native sequence of SARS-CoV-2 nsp2-nsp3 junction. PLpro hydrolyzes the

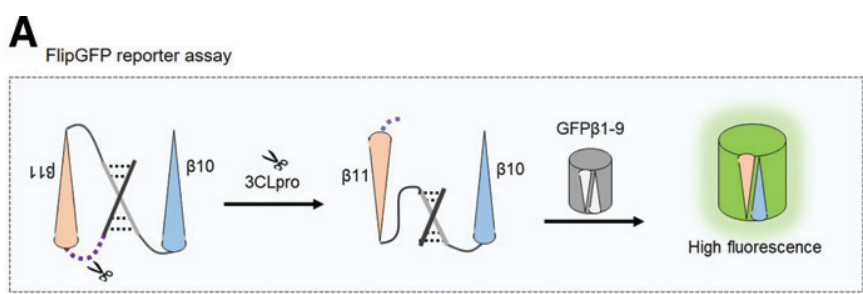

B

NanoBit reporter assay

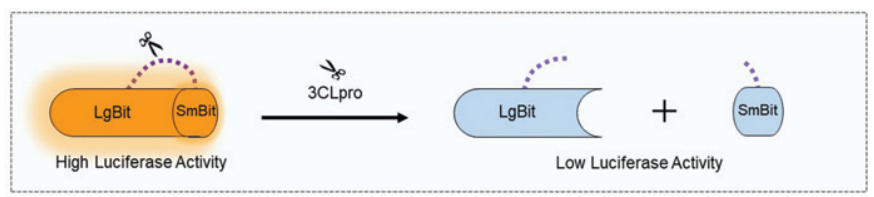

C

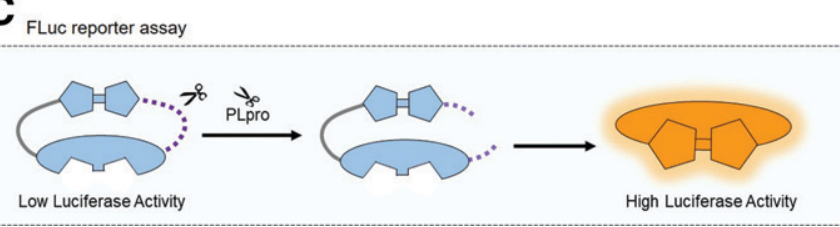

Fig. 3. Design strategies of cell-based assay for SARS-CoV-2 protease. (A) FlipGFP reporter has parallel $\beta 10-11$ strands, which are incompatible with the rest of the GFP fragments. 3CLpro cuts a linker between $\beta 10$ and $\beta 11$ to allow reorientation of $\beta 11$, which results in the formation of fluorescent GFP. (B) NanoBit, a luciferase complementation reporter, has a peptide connecting Large BiT (LgBit) and Small BiT (SmBit) with high luciferase activity. The cleavage of connecting peptide by 3CLpro increases the proximity between LgBit and SmBit, resulting in low luciferase activity. (C) FLuc reporter is inactive as expressed. PLpro cleaves the target peptide to allow FLuc domain dimerization with catalytic activity. 
peptide at the cleavage site that separates the C-terminal fragment and the N-terminal fragment. The two FLuc fragments can then dimerize with the assistance of DnaE to form highly active FLuc. PLpro inhibitors reduce the luminescent signals by suppressing the cleavage of $\mathrm{C}$ - and $\mathrm{N}$-terminal fragments of FLuc, which prevents formation of functional FLuc. In this case, a counterscreen against nonspecific cytotoxicity is essential.

\section{Future Perspectives}

Although clinical trials for protease inhibitors in COVID19 treatment have yet to report significant benefits, previous introduction of protease inhibitors in the treatment of HIV and HCV infections led to dramatic improvements in clinical outcomes. Based on these successful precedents, targeting proteases continues to hold promise as an important therapeutic strategy for COVID-19. 3CLpro is a classic drug target with several approved antiviral drugs for HIV and HCV for which clinical efficacy has been demonstrated. Compared with 3CLpro, research efforts on PLpro have been more limited because of the complexity of this enzyme and difficulties in production of the recombinant enzyme protein. SARS-CoV2 PLpro plays an important role in SARS-CoV-2 replication and suppression of host cell immune response, and thus further elucidation of the PLpro protein structure and mechanism of enzyme reaction will be critical. Potentially, the inhibitor of PLpro may also have a synergistic effect in combination with a 3CLpro inhibitor for COVID-19 treatment.

As viral proteases may share with human host proteases similar substrate motifs and have similar mechanisms for hydrolysis of proteins/peptides, inhibitors of 3CLpro and PLpro could potentially also suppress the activities of critical human proteases. Therefore, it will be important to assess the selectivity of inhibitors to 3CLpro and PLpro during the drug development process from the very beginning of new discovery campaigns. Parallel screens of human proteases such as cathepsin-B and -L with the 3CLpro and PLpro inhibitors during the lead optimization will be particularly important to ensure specificity and selectivity of the final drugs against SARS-CoV-2 proteases (Steuten et al., 2021).

Biochemical enzyme assays play an important role in lead optimization, as they directly measure compound activity on protease enzymes. However, cell-based enzyme assays have become increasingly important for lead optimization, especially for latestage lead compound selection and for further preclinical development in in vivo models. Therefore, both biochemical enzyme assays and cell-based enzyme assays should be developed for lead optimization of protease inhibitors to improve success rates in late-stage preclinical development and future clinical trials.

Finally, a combination therapy of SARS-CoV-2 protease inhibitors with other antiviral agents such as viral entry inhibitors and RdRp inhibitors may greatly improve the therapeutic efficacy for COVID19 treatment, as demonstrated for HIV and HCV treatment.

\section{Acknowledgments}

This work was supported by the Intramural Research Programs of the National Institutes of Health National Center for Advancing Translational Sciences.

\section{Authorship Contributions}

Wrote or contributed to the writing of the manuscript: Zhu, Shyr, Lo, Zheng.

\section{References}

Alexander SPH, Ball JK, and Tsoleridis T (2020) Coronavirus (CoV) proteins (version 2020.5) in the IUPHAR/BPS Guide to Pharmacology Database. IUPHAR/BPS Guide to Pharmacology CITE 2020.

Anand K, Ziebuhr J, Wadhwani P, Mesters JR, and Hilgenfeld R (2003) Coronavirus main proteinase (3CLpro) structure: basis for design of anti-SARS drugs. Science 300:1763-1767.

Báez-Santos YM, St John SE, and Mesecar AD (2015) The SARS-coronavirus papainlike protease: structure, function and inhibition by designed antiviral compounds. Antiviral Res 115:21-38.

Bartenschlager R, Lohmann V, and Penin F (2013) The molecular and structural basis of advanced antiviral therapy for hepatitis $\mathrm{C}$ virus infection. Nat Rev Microbiol 11:482-496.

Berlin DA, Gulick RM, and Martinez FJ (2020) Severe Covid-19. N Engl J Med 383:2451-2460.

Boras B, Jones RM, Anson BJ, Arenson D, Aschenbrenner L, Bakowski MA, Beutler N, Binder J, Chen E, Eng H, et al. (2020) Discovery of a novel inhibitor of coronavirus 3CL protease as a clinical candidate for the potential treatment of COVID-19. bioRxiv:2020.2009.2012.293498

Breidenbach J, Lemke C, Pillaiyar T, Schäkel L, Al Hamwi G, Diett M, Gedschold R, Lopez V, Mirza S, Namasivayam V, et al. (2021) Targeting the main protease of SARS-CoV-2: from the establishment of high throughput screening to the design of tailored inhibitors. Angew Chem Int Ed Engl 60:10423-10429.

Cao B, Wang Y, Wen D, Liu W, Wang J, Fan G, Ruan L, Song B, Cai Y, Wei M, et al. (2020) A trial of lopinavir-ritonavir in adults hospitalized with severe Covid-19. N Engl J Med 382:1787-1799.

Chan KS, Lai ST, Chu CM, Tsui E, Tam CY, Wong MM, Tse MW, Que TL, Peiris JS, Sung J, et al. (2003) Treatment of severe acute respiratory syndrome with lopinavir/ritonavir: a multicentre retrospective matched cohort study. Hong Kong Med $J$ 9:399-406.

Chen S, Jonas F, Shen C, and Hilgenfeld R (2010) Liberation of SARS-CoV main protease from the viral polyprotein: $\mathrm{N}$-terminal autocleavage does not depend on the mature dimerization mode. Protein Cell 1:59-74.

Pan H, Peto R, Henao-Restrepo AM, Preziosi MP, Sathiyamoorthy V, Abdool Karim Q, Alejandria MM, Hernández García C, Kieny MP, Malekzadeh R, et al.; WHO Solidarity Trial Consortium (2021) Repurposed antiviral drugs for Covid-19 interim WHO solidarity trial results. N Engl J Med 384:497-511.

Dai W, Zhang B, Jiang X-M, Su H, Li J, Zhao Y, Xie X, Jin Z, Peng J, Liu F, et al. (2020) Structure-based design of antiviral drug candidates targeting the SARSCoV-2 main protease. Science 368:1331-1335.

De Clercq E (2013) Dancing with chemical formulae of antivirals: A panoramic view (Part 2). Biochem Pharmacol 86:1397-1410.

De Clercq E and Li G (2016) Approved antiviral drugs over the past 50 years. Clin Microbiol Rev 29:695-747.

de Vries M, Mohamed AS, Prescott RA, Valero-Jimenez AM, Desvignes L, O'Connor R, Steppan C, Anderson AS, Binder J, and Dittmann M (2020) Comparative study of a 3CLpro inhibitor and remdesivir against both major SARS-CoV-2 clades in human airway models. bioRxiv:2020.2008.2028.272880.

Dixon AS, Schwinn MK, Hall MP, Zimmerman K, Otto P, Lubben TH, Butler BL, Binkowski BF, Machleidt T, Kirkland TA, et al. (2016) NanoLuc complementation reporter optimized for accurate measurement of protein interactions in cells. ACS Chem Biol 11:400-408.

Froggatt HM, Heaton BE, and Heaton NS (2020) Development of a fluorescencebased, high-throughput SARS-CoV-2 3CL ${ }^{\text {pro }}$ reporter assay. J Virol 94:e1265-20.

Ghosh AK, Osswald HL, and Prato G (2016) Recent progress in the development of HIV-1 protease inhibitors for the treatment of HIV/AIDS. J Med Chem 59:5172-5208.

Gioia M, Ciaccio C, Calligari P, De Simone G, Sbardella D, Tundo G, Fasciglione GF, Di Masi A, Di Pierro D, Bocedi A, et al. (2020) Role of proteolytic enzymes in the COVID-19 infection and promising therapeutic approaches. Biochem Pharmacol 182:114225.

Gordon DE, Jang GM, Bouhaddou M, Xu J, Obernier K, White KM, O'Meara MJ, Rezelj VV, Guo JZ, Swaney DL, et al. (2020) A SARS-CoV-2 protein interaction map reveals targets for drug repurposing. Nature 583:459-468.

Harcourt BH, Jukneliene D, Kanjanahaluethai A, Bechill J, Severson KM, Smith CM, Rota PA, and Baker SC (2004) Identification of severe acute respiratory syndrome coronavirus replicase products and characterization of papain-like protease activity. $J$ Virol 78:13600-13612.

Haugland RP, Yguerabide J, and Stryer L (1969) Dependence of the kinetics of singlet-singlet energy transfer on spectral overlap. Proc Natl Acad Sci USA 63:23-30.

Hoffman RL, Kania RS, Brothers MA, Davies JF, Ferre RA, Gajiwala KS, He M, Hogan RJ, Kozminski K, Li LY, et al. (2020) Discovery of ketone-based covalent inhibitors of coronavirus 3CL proteases for the potential therapeutic treatment of COVID-19. J Med Chem 63:12725-12747.

Hsu M-F, Kuo C-J, Chang K-T, Chang H-C, Chou C-C, Ko T-P, Shr H-L, Chang G-G, Wang AHJ, and Liang P-H (2005) Mechanism of the maturation process of SARSCoV 3CL protease. J Biol Chem 280:31257-31266.

Hurst M and Faulds D (2000) Lopinavir. Drugs 60:1371-1379, discussion 1380-1381.

Huynh T, Cornell W, and Luan B (2021) In silico exploration of inhibitors for SARSCoV-2's papain-like protease. Front Chem 8:624163.

Ivashkiv LB and Donlin LT (2014) Regulation of type I interferon responses. Nat Rev Immunol 14:36-49.

Jamalan M, Barzegari E, and Gholami-Borujeni F (2021) Structure-based screening to discover new inhibitors for papain-like proteinase of SARS-CoV-2: an in silico study. J Proteome Res 20:1015-1026.

Jan J-T, Cheng TR, Juang Y-P, Ma H-H, Wu Y-T, Yang W-B, Cheng C-W, Chen X, Chou T-H, Shie J-J, et al. (2021) Identification of existing pharmaceuticals and herbal medicines as inhibitors of SARS-CoV-2 infection. Proc Natl Acad Sci USA 118:e2021579118. 
Jin Z, Du X, Xu Y, Deng Y, Liu M, Zhao Y, Zhang B, Li X, Zhang L, Peng C, et al. (2020) Structure of $\mathrm{M}^{\text {pro }}$ from SARS-CoV-2 and discovery of its inhibitors. Nature 582:289-293.

Klemm T, Ebert G, Calleja DJ, Allison CC, Richardson LW, Bernardini JP, Lu BGC Kuchel NW, Grohmann C, Shibata Y, et al. (2020) Mechanism and inhibition of the papain-like protease, PLpro, of SARS-CoV-2. EMBO J 39:e106275.

Kuo C-J, Chao T-L, Kao H-C, Tsai Y-M, Liu Y-K, Wang LH-C, Hsieh M-C, Chang S-Y, and Liang P-H (2021) Kinetic characterization and inhibitor screening for the proteases leading to identification of drugs against SARS-CoV-2. Antimicrob Agents Chemother 65:e02577-20.

Kuo C-J, Chi Y-H, Hsu JTA, and Liang P-H (2004) Characterization of SARS main protease and inhibitor assay using a fluorogenic substrate. Biochem Biophys Res Commun 318:862-867.

Lee J, Worrall LJ, Vuckovic M, Rosell FI, Gentile F, Ton A-T, Caveney NA, Ban F, Cherkasov A, Paetzel M, et al. (2020) Crystallographic structure of wild-type SARSCoV-2 main protease acyl-enzyme intermediate with physiological C-terminal autoprocessing site. Nat Commun 11:5877.

Lei J, Kusov Y, and Hilgenfeld R (2018) Nsp3 of coronaviruses: Structures and functions of a large multi-domain protein. Antiviral Res 149:58-74.

Lin C (2006) HCV NS3-4A Serine Protease, in Hepatitis $C$ viruses: genomes and molecular biology, chap 6. Tan SL, ed Horizon Bioscience, Norfolk, UK.

Lo HS, Hui KPY, Lai H-M, He X, Khan KS, Kaur S, Huang J, Li Z, Chan AKN, Cheung HH-Y, et al. (2021) Simeprevir potently suppresses sars-cov-2 replication and synergizes with remdesivir. ACS Cent Sci 7:792-802.

López-Otín C and Matrisian LM (2007) Emerging roles of proteases in tumour suppression. Nat Rev Cancer 7:800-808.

Luan B, Huynh T, Cheng X, Lan G, and Wang H-R (2020) Targeting proteases for treating COVID-19. J Proteome Res 19:4316-4326.

Maiti BK (2020) Can papain-like protease inhibitors halt SARS-CoV-2 replication? ACS Pharmacol Transl Sci 3:1017-1019.

Marras SAE (2006) Selection of Fluorophore and Quencher Pairs for Fluorescent Nucleic Acid Hybridization Probes, in Fluorescent energy transfer nucleic acid probes: designs and protocols (Didenko VV, ed) pp 3-16, Humana Press, Totowa, NJ.

Pathak N, Chen Y-T, Hsu Y-C, Hsu N-Y, Kuo C-J, Tsai HP, Kang J-J, Huang C-H, Chang S-Y, Chang Y-H, et al. (2021) Uncovering flexible active site conformations of SARS-CoV-2 3CL proteases through protease pharmacophore clusters and COVID 19 drug repurposing. ACS Nano 15:857-872.

Pitsillou E, Liang J, Ververis K, Lim KW, Hung A, and Karagiannis TC (2020) Identification of small molecule inhibitors of the deubiquitinating activity of the SARS-CoV-2 papain-like protease: in silico molecular docking studies and in vitro enzymatic activity assay. Front Chem 8:623971.

Qiao J, Li Y-S, Zeng R, Liu F-L, Luo R-H, Huang C, Wang Y-F, Zhang J, Quan B Shen $\mathrm{C}$, et al. (2021) SARS-CoV-2 M $\mathrm{M}^{\text {pro }}$ inhibitors with antiviral activity in a transgenic mouse model. Science 371:1374-1378.

Rathnayake AD, Zheng J, Kim Y, Perera KD, Mackin S, Meyerholz DK, Kashipathy MM, Battaile KP, Lovell S, Perlman S, et al. (2020) 3C-like protease inhibitor block coronavirus replication in vitro and improve survival in MERS-CoV-infected mice. Sci Transl Med 12:eabc5332.

Rawson JMO, Duchon A, Nikolaitchik OA, Pathak VK, and Hu W-S (2021) Development of a cell-based luciferase complementation assay for identification of SARSCoV-2 3CL ${ }^{\text {pro }}$ inhibitors. Viruses 13:173.
Rut W, Groborz K, Zhang L, Sun X, Zmudzinski M, Pawlik B, Wang X, Jochmans D, Neyts J, Młynarski W, et al. (2021) SARS-CoV-2 $\mathrm{M}^{\text {pro }}$ inhibitors and activity-based probes for patient-sample imaging. Nat Chem Biol 17:222-228.

Rut W, Lv Z, Zmudzinski M, Patchett S, Nayak D, Snipas SJ, El Oualid F, Huang TT, Bekes M, Drag M, et al. (2020) Activity profiling and crystal structures of inhibitor-bound SARS-CoV-2 papain-like protease: a framework for anti-COVID-19 drug design. Sci Adv 6:eabd4596.

Shang J, Wan Y, Luo C, Ye G, Geng Q, Auerbach A, and Li F (2020) Cell entry mechanisms of SARS-CoV-2. Proc Natl Acad Sci USA 117:11727-11734.

Shin D, Mukherjee R, Grewe D, Bojkova D, Baek K, Bhattacharya A, Schulz L, Widera M, Mehdipour AR, Tascher G, et al. (2020) Papain-like protease regulates SARS-CoV-2 viral spread and innate immunity. Nature 587:657-662.

Smith E, Davis-Gardner ME, Garcia-Ordonez RD, Nguyen T-T, Hull M, Chen E, Baillargeon P, Scampavia L, Strutzenberg T, Griffin PR, et al. (2020) Highthroughput screening for drugs that inhibit papain-like protease in SARS-CoV-2. SLAS Discov 25:1152-1161.

Steuten K, Kim H, Widen JC, Babin BM, Onguka O, Lovell S, Bolgi O, Cerikan B, Neufeldt CJ, Cortese M, et al. (2021) Challenges for targeting SARS-CoV-2 proteases as a therapeutic strategy for COVID-19. ACS Cent Sci doi.org/10.1021/ acsinfecdis.0c00815.

Wensing AMJ, van Maarseveen NM, and Nijhuis M (2010) Fifteen years of HIV protease inhibitors: raising the barrier to resistance. Antiviral Res 85:59-74.

Yang H, Xie W, Xue X, Yang K, Ma J, Liang W, Zhao Q, Zhou Z, Pei D, Ziebuhr $J$, et al. (2005) Design of wide-spectrum inhibitors targeting coronavirus main proteases. PLoS Biol 3:e324.

Yang H, Yang M, Ding Y, Liu Y, Lou Z, Zhou Z, Sun L, Mo L, Ye S, Pang H, et al. (2003) The crystal structures of severe acute respiratory syndrome virus main protease and its complex with an inhibitor. Proc Natl Acad Sci USA 100: 13190-13195.

Yao T-T, Qian J-D, Zhu W-Y, Wang Y, and Wang G-Q (2020) A systematic review of lopinavir therapy for SARS coronavirus and MERS coronavirus-A possible reference for coronavirus disease-19 treatment option. J Med Virol 92:556-563.

Zhang L, Lin D, Sun X, Curth U, Drosten C, Sauerhering L, Becker S, Rox K, and Hilgenfeld R (2020) Crystal structure of SARS-CoV-2 main protease provides a basis for design of improved $\alpha$-ketoamide inhibitors. Science 368:409-412.

Zhang Q, Schepis A, Huang H, Yang J, Ma W, Torra J, Zhang S-Q, Yang L, Wu H, Nonell S, et al. (2019) Designing a green fluorogenic protease reporter by flipping a beta strand of GFP for imaging apoptosis in animals. J Am Chem Soc 141:4526-4530.

Zhu W, Xu M, Chen CZ, Guo H, Shen M, Hu X, Shinn P, Klumpp-Thomas C, Michael SG, and Zheng W (2020) Identification of SARS-CoV-2 3CL protease inhibitors by a quantitative high-throughput screening. ACS Pharmacol Transl Sci 3:1008-1016.

Address correspondence to: Dr. Wei Zheng, National Center for Advancing Translational Sciences, National Institutes of Health, 9800 Medical Center Dr., MSC: 3375, Bethesda, Maryland 20854. E-mail: wzheng@mail.nih.gov. 